\title{
LOCALIZATION OF PERIODIC ORBITS \\ OF AUTONOMOUS SYSTEMS BASED ON HIGH-ORDER EXTREMUM CONDITIONS
}

\author{
KONSTANTIN E. STARKOV
}

Received 12 November 2003 and in revised form 16 March 2004

This paper gives localization and nonexistence conditions of periodic orbits in some subsets of the state space. Mainly, our approach is based on high-order extremum conditions, on high-order tangency conditions of a nonsingular solution of a polynomial system with an algebraic surface, and on some ideas related to algebraically-dependent polynomials. Examples of the localization analysis of periodic orbits are presented including the Blasius equations, the generalized mass action (GMA) system, and the mathematical model of the chemical reaction with autocatalytic step.

\section{Introduction}

The study of periodic orbits has a significant impact on understanding the dynamics of multidimensional systems. Apart from its purely theoretical importance, this research area has various engineering applications, for example, in chemical engineering, electrical engineering, and so forth. One of the interesting problems of qualitative theory of ordinary differential equations

$$
\dot{x}=f(x)
$$

with $x=\left(x_{1}, \ldots, x_{n}\right)^{T} \in \mathbb{R}^{n}, f(x)=\left(f_{1}(x), \ldots, f_{n}(x)\right)^{T} \in C^{\infty}\left(\mathbb{R}^{n}\right)$, consists in finding a domain in the state space containing all periodic orbits. It is well known that this problem is difficult, for example, in the case of a search for unstable periodic orbits of multidimensional systems (1.1). Though many papers devoted to this topic for two-dimensional systems have been published, see, for example, the references in $[18,30]$, one can give only a short list of publications concerning periodic orbits of three- and higher-dimensional systems. Here, one can recall the papers $[1,2,4,7,8,10,11,14,22,23,24,27]$. It is worthwhile to mention that the localization of periodic orbits is closely related to the similar problem for attractors. The idea of the characterization of a chaotic attractor $\mathbf{A}$ by means of infinitely many unstable periodic orbits embedded in $\mathbf{A}$ has been used in a number of articles, see, for example, [5] concerning the Lorenz system and the references therein. 
Thus the localization of domains with periodic orbits can provide information respecting a location of domains with chaotic attractors.

Let $h \in C^{\infty}\left(\mathbb{R}^{n}\right)$ and $L_{f} h$ be the Lie derivative of $h$ along the vector field $f$. Also, $L_{f}^{i} h=$ $L_{f}\left(L_{f}^{i-1} h\right), i \geq 1, L_{f}^{0} h=h$. The function $h$ will be used in the solution of the localization problem. If it is not specified explicitly, we assume that $f$ is a polynomial vector field and $h$ is a polynomial. The main objective of this paper is to find some sets $K$ in $\mathbb{R}^{n}$ in terms of conditions imposed on $f$ and $h$ such that (1) any periodic orbit of (1.1) is contained in $K$, with $K$ a domain, or (2) any periodic orbit has common points with $K$; here $K$ is a surface. Further, by using these sets, we propose nonexistence conditions of periodic orbits in some domains of the state space. Here, by saying that a periodic orbit $\Gamma$ is not contained in some set $U$, we mean that $\Gamma$ is not totally contained in $U$.

This paper is the extended version of the short conference paper [28].

The structure of this paper is as follows. Section 2 presents the main localization/ nonexistence result of periodic orbits and some relevant assertions. In Section 3, we are concerned with the relationship between invariant sets and periodic orbits. The localization of periodic orbits for a class of systems (1.1) satisfying one triangularizability condition is discussed in Section 4. Section 5 contains a refinement of the main result under one condition expressed in terms of equilibrium points. In Section 6, we address applications of our results to the Blasius equations, the generalized mass action (GMA) system, and the mathematical model of the chemical reaction with autocatalytic step. General comments respecting a solution of the localization problem are contained in Section 7. Section 8 includes our concluding remarks.

\section{The main result and some corollaries}

By $\varphi(x, t)$ we denote a solution of system $(1.1)$ such that $\varphi(x, 0)=x$. Let $f_{s}, s=1, \ldots, n$, and $h \in C^{\infty}\left(\mathbb{R}^{n}\right)$. We introduce

(1) sets $K_{1}=\left\{x \in \mathbb{R}^{n} \mid L_{f} h(x)=0\right\}, K_{1}^{+}=\left\{x \in \mathbb{R}^{n} \mid L_{f} h(x)>0\right\}, K_{1}^{-}=\left\{x \in \mathbb{R}^{n} \mid\right.$ $\left.L_{f} h(x)<0\right\}$

(2) sets $K_{j}$ defined with the help of the following system of equations:

$$
L_{f}^{i} h(x)=0, \quad i=1, \ldots, j
$$

(3) sets $K_{k}^{+}\left(K_{k}^{-}\right)$defined with the help of (2.1) for $j=k-1$ and one inequality $L_{f}^{k} h(x)>0\left(L_{f}^{k} h(x)<0\right.$ appropriately $) ; K_{k}^{ \pm}=K_{k}^{+} \cup K_{k}^{-}, k \geq 2 ;$

(4) sets

$$
\begin{gathered}
S_{k}^{+}=K_{2}^{+} \cup K_{3}^{ \pm} \cup \cdots \cup K_{2 j-1}^{ \pm} \cup K_{2 j}^{+}, \quad k=2 j, \\
S_{k}^{+}=K_{2}^{+} \cup K_{3}^{ \pm} \cup \cdots \cup K_{2 j-1}^{ \pm} \cup K_{2 j}^{+} \cup K_{2 j+1}^{ \pm}, \quad k=2 j+1 .
\end{gathered}
$$

Similarly, sets $S_{k}^{-}$are defined with the help of a simultaneous replacement of all "+” by "-" in (2.2). Though all sets introduced here depend on $h$, this dependence will be usually suppressed.

Further, by $C\{V\}$ we denote the complement of the set $V$ in the space $\mathbb{R}^{n}$. If $g$ is some function of $n$ real variables, then we denote by $\left.g\right|_{\{V\}}$ the restriction of $g$ on $V$. Let $S$ be any 
set in $\mathbb{R}^{n}$. Then by $\operatorname{Inv}(S)$ we denote the maximal (with respect to the inclusion) invariant subset of $S$ of the phase flow of system (1.1).

It follows from the construction of sets $K_{s}$ and local analyticity of the solution that

$$
K_{1} \supset K_{2} \supset \cdots \supset K_{s} \supset \cdots \supset f^{-1}(0)
$$

and $\cap_{s=1}^{\infty} K_{s}=\operatorname{Inv} K_{1}$.

Further, the following fact plays the key role.

Lemma 2.1. Let $f$ in (1.1) and $h \in C^{\infty}\left(\mathbb{R}^{n}\right)$. If the point $x_{0}$ is contained in the periodic orbit $\Gamma$ and $h\left(\varphi\left(x_{0}, t\right)\right)$ is not constant as a time function, then there are, at least, two instants $t_{\max }, t_{\min } \geq 0$ and positive integers $k_{1}$ and $k_{2}$ such that $\varphi\left(x_{0}, t_{\min }\right) \in K_{2 k_{1}}^{+}$and $\varphi\left(x_{0}, t_{\max }\right) \in$ $K_{2 k_{2}}^{-}$.

Proof. We notice that $h\left(\varphi\left(x_{0}, t\right)\right)$ is a periodic time function with a positive period. Therefore one can find instants $t_{\max }$ and $t_{\min }$ such that

$$
\begin{aligned}
& h\left(\varphi\left(x_{0}, t_{\max }\right)\right)=\max _{t} h\left(\varphi\left(x_{0}, t\right)\right), \\
& h\left(\varphi\left(x_{0}, t_{\min }\right)\right)=\min _{t} h\left(\varphi\left(x_{0}, t\right)\right) .
\end{aligned}
$$

By applying high-order extremum conditions, we obtain the desirable conclusion.

Lemma 2.1 entails the following proposition.

Proposition 2.2. Let $f_{s}, s=1, \ldots, n$, and $h \in C^{\infty}\left(\mathbb{R}^{n}\right)$. If the set $\operatorname{Inv}\left(K_{1}\right)=K_{1}$, then all periodic orbits are contained in $K_{1}$.

Proof. By Lemma 2.1, each periodic orbit has common points with $K_{1}$. The latter implies the desirable result because of the invariance of $K_{1}$.

For example, we consider the system $\dot{x}=A_{0} x+g(x) \cdot A_{1} x$. Assume that there is a vector row $C$ such that $C A_{1}=0$ and $C$ is a right eigenvector of $A_{0}$ corresponding to some nonzero real eigenvalue. We take the function $h(x)=C x$. Then $\operatorname{Ker} C=K_{1}$ and $K_{1}$ is the invariant subspace containing all periodic orbits.

We define the following sets: $M_{1}=\operatorname{Inv}\left(K_{1}\right) \cap C\left\{f^{-1}(0)\right\}$ and $M_{2}=K_{1}-\operatorname{Inv}\left(K_{1}\right)$. Now we are in position to state the following theorem.

Theorem 2.3. Let $f$ and $h \in C^{\infty}\left(\mathbb{R}^{n}\right)$. Assume that the set $K_{1}$ is a union of a finite number of infinitely differentiable hypersurfaces in $\mathbb{R}^{n}$. If $\Gamma$ is a periodic orbit, then one of the following cases is realized: (1) $\Gamma \subset M_{1} ;(2) \Gamma \cap \operatorname{Inv}\left(K_{1}\right)=\varnothing, \Gamma \cap S_{1}^{+} \neq \varnothing, \Gamma \cap S_{1}^{-} \neq \varnothing$, and

$$
\operatorname{card}\left\{\Gamma \cap\left(\cup_{s=1}^{\infty} K_{2 s}^{+}\right)\right\}=\operatorname{card}\left\{\Gamma \cap\left(\cup_{s=1}^{\infty} K_{2 s}^{-}\right)\right\}>0 .
$$

In addition, suppose that $f$ and $h$ are polynomials. Let $\alpha=\max _{s=1, \ldots, n} \operatorname{deg} f_{s} \geq 2, \operatorname{deg} h=$ $p, \max (p ; n) \leq \beta$, and

$$
N=\frac{1}{2} \sum_{k=0}^{n}(2 \beta+2 k(p-1))^{2 n+2} .
$$


Then the formula

$$
\operatorname{card}\left\{\Gamma \cap\left(\cup_{2 s \leq N} K_{2 s}^{+}\right)\right\}=\operatorname{card}\left\{\Gamma \cap\left(\cup_{2 s \leq N} K_{2 s}^{-}\right)\right\}>0
$$

is valid.

Proof. Firstly, we note that $M_{1}$ is invariant. Suppose that $\Gamma$ is not contained in the set $M_{1}$. Then $\Gamma$ is contained in the set $C\left\{\operatorname{Inv}\left(K_{1}\right)\right\}$. It is easy to see that $h(\varphi(z, t))$ is not constant as a time function for any point $z \in C\left\{\operatorname{Inv}\left(K_{1}\right)\right\}$. We take $x \in \Gamma$. Then the period $T$ of the function $L_{f} h(\varphi(x, t))$ is a positive number. We can meet the following cases of the location of $\Gamma:(1) \Gamma \subset\left(\left(L_{f} h\right)^{-1}((0, \infty)) \cup\left(L_{f} h\right)^{-1}((-\infty, 0))\right)$, (2) $\Gamma$ has exactly one common point with the set $M_{2}$, (3) $\Gamma$ intersects the set $M_{2}$ in a few points.

(1) Firstly, we assume that

$$
\Gamma \subset\left(L_{f} h\right)^{-1}((-\infty, 0))
$$

and take $x_{0} \in \Gamma$. Let $T>0$ be a minimal period of $\Gamma$. Since $\Gamma$ is compact, we can replace $h$ by $h+c$, where $c$ is a constant such that $h+c$ is nonnegative on $\Gamma$. So $h+c$ is a Lyapunov function on $\Gamma$. Besides, the restriction $\left.L_{f} h\right|_{\Gamma}$ is bounded above and below. Therefore we can apply the LaSalle theorem [13, Theorem 1] to $\Gamma$. As a result, we obtain that the distance of points $\varphi\left(x_{0}, t_{m}\right)$ from the set $S_{1}$ vanishes as $t_{m} \rightarrow \infty, m \rightarrow \infty$. By taking $t_{m}=m T$, we deduce that $x_{0} \in S_{1}$. This contradicts (2.8). Now we assume that $\Gamma \subset\left(L_{f} h\right)^{-1}((0, \infty))$. Since the change of time $t \rightarrow-t$ preserves periodic orbits, we obtain that this case is examined in the same way.

(2) Here, by using necessary extremum conditions applied to the time function $h\left(\varphi\left(x_{0}\right.\right.$, $t)$ ), we get that $\Gamma$ has at least two common points with the set $S_{1}$. So this case is not possible.

(3) Consider the third case. Assume that the function $h\left(\varphi\left(x_{0}, t\right)\right)$ has no local extremum at the point $t=0$ (we can get $h\left(\varphi\left(x_{0}, t\right)\right.$ ) with this property by a small time shift if it is necessary). We notice that the function $L_{f} h\left(\varphi\left(x_{0}, t\right)\right)$ has the same period $T$ just as $h\left(\varphi\left(x_{0}, t\right)\right)$. We take the function $L_{f} h(\varphi(x, t))$ restricted on the open interval $(0, T)$. Then it follows from Pólya and Szegó [20, Example 22, page 39] that $L_{f} h\left(\varphi\left(x_{0}, t\right)\right)$ has an even number of sign reversals on $(0, T)$. Now, by using sign extremum conditions applied to each of the points of sign reversals, we establish that there is an equal number of minimum points and maximum points of the function $h(\varphi(x, t))$ restricted on $(0, T)$. Therefore, by applying high-order extremum conditions to each of these points, we derive (2.5).

It remains to consider the polynomial case of $(f, h)$. We use the Gabrielov-Khovanskii theorem, see [6, Theorem 3]. Namely, assume that $x$ is not an equilibrium point. Then $h(\varphi(x, t))$ is not constant as a time function if and only if there is an integer $i$ not exceeding $N$ given in formula (2.6) for which $L_{f}^{i} h(x) \neq 0$.

So, by using this theorem, we conclude that $K_{i}=f^{-1}(0)$ for $i>2 N$, which entails $K_{2 s}^{ \pm}=$ $\varnothing$ with $s>N$. As a result, we come to formula (2.7).

Theorem 2.3 is a generalization of the classical result of Poincaré concerning tangential curves, see, for example, [30, Theorem 1.9]. Indeed, let $n=2$ and let $U$ be a domain 
in $\mathbb{R}^{2}$ in which $L_{f} h \leq 0$ or $L_{f} h \geq 0$. Assume that $K_{1} \cap U$ does not contain any periodic orbit. By Theorem 2.3, the unique possibility for $\Gamma$ to be located in $U$ is to intersect both of the sets $U \cap K_{1}^{+}$and $U \cap K_{1}^{-}$. But this leads to a contradiction since the function $L_{f} h$ is sign-definite in $U$.

Let $f_{s}, s=1, \ldots, n$, and $h \in C^{\infty}\left(\mathbb{R}^{n}\right)$. Now we come to a formulation of nonexistence conditions of periodic orbits which are based on the algebraic relationship between $h$, $L_{f} h$, and $L_{f}^{2} h$.

Corollary 2.4. Let $L_{f} h(x)=a+g_{1}(x) h(x)$ with a constant $a \neq 0$. In addition, assume that (1) $\left.L_{f}^{2} h\right|_{\{h(x)>0\}}>0$ and $\left.L_{f}^{2} h\right|_{\{h(x)<0\}}<0$, or (2) $\left.L_{f}^{2} h\right|_{\{h(x)<0\}}>0$ and $\left.L_{f}^{2} h\right|_{\{h(x)>0\}}<0$. Then system (1.1) has no periodic orbits.

Proof. Indeed, in these cases, the surface $h(x)=0$ is permeable only in one direction by the phase flow. So each periodic orbit $\Gamma \subset\{h(x)>0\}$ or $\Gamma \subset\{h(x)<0\}$. Therefore one of sets $K_{2}^{+}$and $K_{2}^{-}$is empty, which entails the desirable result.

Corollary 2.5. Let $L_{f}^{2} h(x)=a+g_{2}(x) L_{f} h(x)+\operatorname{sgn}(a) g_{3}(x)$ with a constant $a \neq 0$ and $a$ nonnegative function $g_{3}$ on $\mathbb{R}^{n}$. Then system (1.1) has no periodic orbits.

Proof. Analogously, one of the sets $K_{2}^{+}$and $K_{2}^{-}$is empty, which entails the desirable result.

We present one sufficient nonexistence condition of periodic orbits stated in terms of sets $S_{2 N}^{+}, S_{2 N}^{-}$.

Proposition 2.6. Let $f_{s}, s=1, \ldots, n$, and $h \in C^{\infty}\left(\mathbb{R}^{n}\right)$. Assume that the function $h$ is chosen in such a way that $h(\varphi(x, t))$ is not constant as a time function for any point $x \notin f^{-1}(0)$. Besides, suppose that one of the following conditions is realized: (1) $S_{2 N}^{+} \cup f^{-1}(0)=K_{1}$; (2) $S_{2 N}^{-} \cup f^{-1}(0)=K_{1}$. Then system (1.1) has no periodic orbits.

Proof. Let $x_{0} \in \Gamma$, where $\Gamma$ is a periodic orbit. Assume that condition (1) or condition (2) holds. Then the function $h\left(\varphi\left(x_{0}, t\right)\right)$ has no minimum points (no maximum points) appropriately. This contradicts Lemma 2.1.

As an illustrating example, we consider system (1.1) with $n=2$ and $f_{1}\left(x_{1}, x_{2}\right)=a_{1} x_{1}+$ $a_{2} x_{2}, f_{2}\left(x_{1}, x_{2}\right)=p\left(x_{1}, x_{2}\right)$. Here, $p$ is a homogeneous form of even degree. Let $a_{2} \neq 0$ and $p\left(1,-a_{1} a_{2}^{-1}\right)>0$. Then this system has no periodic orbits. Indeed, we take $h(x)=x_{1}$ and compute that $K_{2}^{+} \cup(0,0)=K_{1}$.

Also, we note that if $K_{1}=f^{-1}(0)$, then system (1.1) has no periodic orbits. Here, the classical example is a gradient system. The case $K_{n}=f^{-1}(0)$ will be discussed below.

\section{Invariant sets and the localization of periodic orbits}

In this section, we study the set $\operatorname{Inv}\left(K_{1}\right)$ and present new localization conditions of periodic orbits.

Consider the equality

$$
L_{f}^{m+1} h(x)=\sum_{s=1}^{m} a_{s}(x) L_{f}^{s} h(x)
$$


where $a_{s}(x), s=1, \ldots, m$, are rational functions, that is, $a_{s}=p_{s} / q_{s}$, with all $p_{s}, q_{s}$ polynomials. In addition, we suppose that $m$ is minimal with this property. Let $G$ be a set of zeroes of the polynomial $\prod_{j=1}^{m} q(x)$. Firstly, we give the following lemma.

Lemma 3.1. If (3.1) is valid, then $K_{m} \cap C\{G\} \subset \operatorname{Inv} K_{1}$.

Proof. We take any point $x \in K_{m} \cap C\{G\}$. It follows from (3.1) that $L_{f}^{m+1} h(x)=0$. Now, by applying iteratively the Lie operator $L_{f}$ to formula (3.1) and making substitutions of equalities (3.1) into expressions for $L_{f}^{s} h$, with $s \geq m+2$, we come to the formula $L_{f}^{m+j} h(x)=\sum_{s=1}^{m} a_{s j}(x) L_{f}^{s} h(x)=0, j=2,3, \ldots$, in which all $a_{s j}$ are some rational functions with denominators having zeroes in the set $G$. Since the solution $\varphi$ is locally analytic, the latter formula entails the desirable result.

Obviously, if all $a_{s}$ are polynomials in (3.1), then Inv $K_{1}=K_{m}$.

We address the case of $m=1$ in (3.1), where we are concerned with its relation with classical results. We recall one definition.

Definition 3.2. Suppose that there exists a polynomial $h$ of $n$ variables satisfying the equality

$$
L_{f} h(x)=a_{0} h(x)
$$

for some polynomial $a_{0}$ of $n$ variables. Then $h$ is called the Darboux polynomial and $a_{0}$ is called a cofactor, see, for example, [15].

It is well known that these polynomials are useful in studying integrability property of polynomial systems, and up to now, there have been published many results respecting finding Darboux polynomials for the Lorenz system [17], the Rikitake system [15], the Rössler system [16], and so forth. Our new example is the Sprott system [25, Example K, Table 11] $\dot{x}_{1}=x_{1} x_{2}-x_{3}, \dot{x}_{2}=x_{1}-x_{2}, \dot{x}_{3}=x_{1}+a x_{3}$, in which the coefficient 0.3 is replaced by a nonzero parameter $a$. We have found by the method of indefinite coefficients that the list of all quadratic Darboux polynomials consists of two polynomials, $h(x)=x_{1}-x_{2}^{2} / 2-$ $2 x_{3}$ for $a=-5 / 2$ and $h(x)=\left(x_{2}-x_{3}\right)^{2}$ for $a=-1$. The latter means that all periodic orbits are located in the set $x_{1}-x_{2}^{2} / 2-2 x_{3}=0$ for $a=-5 / 2$ and in the plane $x_{2}-x_{3}=0$ for $a=-1$.

Below, we state the relationship between Darboux polynomials and the formula for $\operatorname{Inv} K_{1}$.

Lemмa 3.3. If $h$ is a Darboux polynomial with a cofactor $a_{0}$ for system (1.1), then

$$
\operatorname{Inv} K_{1} \supset K_{1} \cap C\left\{a_{0}^{-1}(0)\right\} .
$$

Proof. We compute

$$
L_{f}^{2} h=L_{f} h \cdot\left(a_{0}^{-1} L_{f} a_{0}+a_{0}\right)
$$

Let $b_{2}=L_{f} a_{0}+a_{0}^{2}$. By applying $L_{f}$ to this formula and using (3.4), we get the similar formula for $L_{f}^{3} h: L_{f}^{3} h=a_{0}^{-2} b_{3} L_{f} h$ for some polynomial $b_{3}$, and so forth. As a result, there 
is a sequence of polynomials $\left\{b_{s}\right\}$ such that $L_{f}^{s} h=b_{s} a_{0}^{1-s} L_{f} h, s=2, \ldots, \infty$. After this, we argue as above.

If $a_{0}$ is constant in (3.2), then Inv $K_{1}=K_{1}$.

In the general case, we meet the problem of finding $h$ satisfying (3.1) for some natural number $m$ and functional coefficients $a_{s}$. This problem is difficult and leads to a solution of a partial differential equation of a high unknown degree. Instead of going in this direction, we choose another way related to results of the author which are based on using algebraically-dependent polynomials, see [26].

We take a polynomial $h$ of $n$ real variables and then form a sequence of polynomials $\left\{L_{f}^{s} h\right\}, s=1,2, \ldots$ It follows from the Perron theorem, see the modern version in [19, Theorem 3.1], that there is a natural number $m$ such that the polynomials $\left\{L_{f}^{s} h\right\}, s=$ $1, \ldots, m$, are algebraically independent while the polynomials $\left\{L_{f}^{s} h\right\}, s=1, \ldots, m+1$, are algebraically dependent, with $m \leq n$. This means that one can find a nonzero polynomial $\Phi\left(w_{1}, \ldots, w_{m+1}\right)$ such that

$$
\Phi\left(L_{f}^{s} h(x), s=1, \ldots, m+1\right) \equiv 0 .
$$

Here, without loss of generality, one can assume additionally that the degree $\operatorname{deg} \Phi(w)$ is minimal with this property. Applying the Lie derivative $L_{f}$ to (3.5), we get the identity

$$
L_{f}^{m+2} h(x) \cdot q_{m+2}\left(L_{f}^{s} h(x), s=1, \ldots, m+1\right)+p\left(L_{f}^{s} h(x), s=1, \ldots, m+1\right) \equiv 0,
$$

where $p, q_{m+2}$ are two polynomials of $m+1$ real variables $w=\left(w_{1}, \ldots, w_{m+1}\right)$. It is proved, see [26, Lemma 1], that $\operatorname{deg} q_{m+2}(w)<\operatorname{deg} \Phi(w)$ and $q_{m+2}\left(L_{f}^{s} h(x), s=1, \ldots, m+1\right)$ is a nonzero polynomial. So the set $S:=\left\{x \in \mathbb{R}^{n} \mid q_{m+2}\left(L_{f}^{s} h(x), s=1, \ldots, m+1\right)=0\right\}$ is a proper subset in $\mathbb{R}^{n}$, that is, $S \varsubsetneqq \mathbb{R}^{n}$. Then we apply $L_{f}$ to (3.6), and so forth.

Our next result shows explicitly the location of $\operatorname{Inv}\left(K_{1}\right)$ in the polynomial case. Namely, we have the following theorem.

Theorem 3.4. Define the set

$$
\begin{gathered}
M_{1}=\left\{x \in \mathbb{R}^{n} \mid L_{f}^{s} h(x)=0, s=1, \ldots, m+1,\right. \\
\left.q_{m+2}\left(L_{f}^{s} h(x), s=1, \ldots, m+1\right) \neq 0\right\} .
\end{gathered}
$$

Then $M_{1} \subset \operatorname{Inv}\left(K_{1}\right) \subset K_{n+1}$.

Proof. By computations taken from [26, Lemma 1], we obtain that $L_{f}^{m+j} h(x)$ is expressed as a rational function due to (3.6) for some polynomial $q_{m+j}$, while its denominator has the same zero set $S$ for any positive integer $j$. Now let $L_{f}^{s} h\left(x_{*}\right)=0, s=1, \ldots, m+1$ and $x_{*} \notin S$. Then $L_{f}^{s} h\left(x_{*}\right)=0$ for any $s=m+2, \ldots, \infty$. Since the solution of (1.1) is locally analytic, then by using the theorem on continuation of a solution, we get that $L_{f} h\left(\varphi\left(x_{*}, t\right)\right)=0$ for any $t$ from the maximal interval of the existence of the solution. Now let $x_{*} \in \operatorname{Inv}\left(K_{1}\right)$. Then, by local analyticity of a solution, we conclude that $L_{f}^{i} h\left(x_{*}\right)=0$ for $i=1, \ldots, m+1$ and $x_{*} \in K_{m+1}$. 
To summarize, there are the following cases of locating periodic orbits inside the set $C\{S\}$ : (1) each periodic orbit $\Gamma$ is contained in the set $K_{m+1}$, or (2) $\Gamma$ is not contained in $K_{1}$, but $\Gamma$ has common points with both of the sets $K_{1}^{+}$and $K_{1}^{-}$and, besides, card $\{\Gamma \cap$ $\left.\left(\cup_{2 s<m+1} K_{2 s}^{+}\right)\right\}=\operatorname{card}\left\{\Gamma \cap\left(\cup_{2 s<m+1} K_{2 s}^{-}\right)\right\}>0$. By applying the ideas of Proposition 2.6 in this situation, if $S_{m+1}^{+} \cup f^{-1}(0)=K_{1}$ or $S_{m+1}^{-} \cup f^{-1}(0)=K_{1}$, then the system (1.1) has no periodic orbits contained in the set $C\{S\}$.

\section{Periodic orbits of triangularizable systems}

In this section, we describe new localization conditions of periodic orbits which are based on Proposition 2.6. Suppose that the vector field $f$ is analytic and $f(0)=0$. Then $f$ can be written as $f(x)=A(x) x$ for some analytic matrix-valued function $A: \mathbb{R}^{n} \rightarrow \mathbb{R}^{n \times n}$, or as $f(x)=\sum_{j \in J} x^{j} A_{j} x$ in the standard multinomial notation. By $Z$ we denote the set $\left\{x \in \mathbb{R}^{n} \mid \operatorname{det} A(x)=0\right\}$. Assume that the set of matrices $\left\{A_{j} \mid j \in J\right\}$ is triangularizable. This means that there is a basis of $\mathbb{R}^{n}$ such that the matrix $A(x)$ is upper triangular. We mention here that many results concerning triangularizability conditions of the set of matrices $\left\{A_{j} \mid j \in J\right\}$ are contained in [21]. Now we are in position to state the following proposition.

Proposition 4.1. Suppose that the matrix $A(x)$ is given in the upper triangular form and the set $Z$ is invariant. Then all periodic orbits of system (1.1) are located in $Z$.

Proof. By $\mathscr{S}_{j}$ we denote the system obtained from (1.1) by taking the restriction of (1.1) on the plane $\Pi_{j}: x_{j}=\cdots=x_{n}=0$. It follows from the triangular structure of the matrix $A(x)$ that $\Pi_{j} \cup\left\{a_{j j}\left(x_{1}, \ldots, x_{j-1}, 0, \ldots, 0\right)=0 \mid\left(x_{1}, \ldots, x_{j-1}\right)^{T} \in \mathbb{R}^{j-1}\right\}$ is the invariant set of the system $\mathscr{Y}_{j}$. Now, with the help of Proposition 2.6 successively applied to systems (1.1), $\mathscr{S}_{j}, j=n, n-1, \ldots, 2$, we get that periodic orbits are not contained in $\Pi_{j}, j=1,2, \ldots, n$.

Hence, since $\operatorname{det} A(x)=\prod_{j=1}^{n} a_{j j}(x)$ and $Z$ is invariant, we get that any periodic orbit is located in the set $Z$.

For example, suppose that the matrix $A(x)$ is upper triangular with constant diagonal elements. Applying the last result to system (1.1), we get that, in this case, system (1.1) has no periodic orbits.

Now we take the three-dimensional system with the upper triangular matrix $A(x)$ :

$$
\begin{gathered}
\dot{x}_{1}=a_{11}\left(x_{1}, x_{2}, x_{3}\right) x_{1}+a_{12}\left(x_{1}, x_{2}, x_{3}\right) x_{2}+a_{13}\left(x_{1}, x_{2}, x_{3}\right) x_{3}, \\
\dot{x}_{2}=a_{22}\left(x_{1}, x_{2}, x_{3}\right) x_{2}+a_{23}\left(x_{1}, x_{2}, x_{3}\right) x_{3}, \\
\dot{x}_{3}=a_{33}\left(x_{1}, x_{2}, x_{3}\right) x_{3},
\end{gathered}
$$

and take the function $h(x)=x_{3}$. By $A_{33}$ we denote the set defined by the equation $a_{33}(x)=$ 0 . Below, by $\mathscr{P}_{i}$, we denote the hyperplane $x_{i}=0$ in $\mathbb{R}^{3}$. We notice that the hyperplane $\mathscr{P}_{3}$ is the invariant set and $S_{1}=\mathscr{P}_{3} \cup A_{33}$. Assume that $L_{f} a_{33}(x)>0$ for $x_{3} \neq 0$. We take any periodic orbit $\Gamma$ outside $\mathscr{P}_{3}$. Then the function $\left.L_{f} a_{33}\right|_{\{\Gamma\}}$ cannot get different signs since $\Gamma$ cannot cross $\mathscr{P}_{3}$. Thus $\left.L_{f}^{2} h\right|_{\{\Gamma\}}$ cannot change its sign. As a result, there are no periodic 
orbits outside $\mathscr{P}_{3}$. So it remains to study periodic orbits of the two-dimensional system

$$
\begin{gathered}
\dot{x}_{1}=a_{11}\left(x_{1}, x_{2}, 0\right) x_{1}+a_{12}\left(x_{1}, x_{2}, 0\right) x_{2}, \\
\dot{x}_{2}=a_{22}\left(x_{1}, x_{2}, 0\right) x_{2},
\end{gathered}
$$

which can be fulfilled by any of the known methods of the study of periodic orbits of two-dimensional systems.

\section{The reduction of the main result in the case of $K_{n}=f^{-1}(0)$}

Below, our goal is to find conditions leading to $K_{n}=f^{-1}(0)$. In this case, formula (2.5) is reduced to $\operatorname{card}\left\{\Gamma \cap\left(\cup_{2 s \leq n} K_{2 s}^{+}\right)\right\}=\operatorname{card}\left\{\Gamma \cap\left(\cup_{2 s \leq n} K_{2 s}^{-}\right)\right\}>0$. This allows us to localize the position of periodic orbits more efficiently. Firstly, we notice that

$$
\left(L_{f}^{s} h(x), s=1, \ldots, n\right)^{T}=T(x) f(x)
$$

with $T(x)$ a polynomial matrix. We take $x_{0} \in K_{n}$. Then it follows from (5.1) that $x_{0} \in$ $f^{-1}(0)$, provided the determinant $\operatorname{det} T\left(x_{0}\right) \neq 0$. Hence, we obtain that

$$
K_{n} \cap\{x \mid \operatorname{det} T(x) \neq 0\} \subset f^{-1}(0) .
$$

The last formula will be applied to a polynomial system with $n-1$ linear components $f_{i}$, $i=1, \ldots, n-1 ; f_{n}$ is a nonlinear polynomial.

We recall two classical definitions borrowed from control theory. Consider a linear control system $\dot{w}=K w+B u$ with a solution $\gamma$ and an observation function $y=C w$. Here, $K$ is a constant $n \times n$ matrix, $C$ is a constant vector row. The pair $(K, C)$ is called observable if $C \exp (K t) w$ is not equal to zero as a time function for any nonzero state vector $w$. The minimal number $i$ for which $\partial^{i} C \gamma / \partial t^{i}$ depends on a control $u$ explicitly is called the relative degree of the triple $(K, B, C)$. We establish the following lemma.

Lemma 5.1. Let $A=\left(a_{i j}\right)_{i, j=1, \ldots, n}$ be an $n \times n$ matrix representing the linear part of $f$ and let $A_{n-1}$ be an $(n-1) \times(n-1)$ submatrix got from $A$ by deleting the last column and the last row. Suppose that (1) $a_{n-1 n} \neq 0$, and (2) the relative degree of the triple $\left(A_{n-1}, l, C\right)$, with $l=\left(0, \ldots, 0, a_{n-1 n}\right)^{T} \in \mathbb{R}^{n-1}$, is equal to $n-1$. Take the function $h(x)=C\left(x_{1}, \ldots, x_{n-1}\right)^{T}$. Let the pair $\left(A_{n-1}, C\right)$ be observable. Introduce the matrix $T(x)$ by formula (5.1) for this choice of h. Then $K_{n}=f^{-1}(0)$.

Proof. Indeed, since $L_{f}^{s} h(x)=C A^{s} x, s \leq n-1$, we deduce from the observability rank condition that $T$ is a constant nonsingular matrix. Therefore $K_{n}=f^{-1}(0)$.

For example, the Rössler system with the function $h(x)=x_{2}$ satisfies the conditions of Lemma 5.1 with

$$
T=\left(\begin{array}{ccc}
0 & 1 & 0 \\
1 & a & 0 \\
a & a^{2}-1 & -1
\end{array}\right)
$$


$a$ is a parameter of the Rössler system. Some of the chaotic Sprott systems [25] with one nonlinear monomial term satisfy the conditions of the last lemma for a proper choice of $h$.

\section{Applications}

Now we demonstrate how the results stated above can work.

(1) We consider the Blasius equations taken from the theory of fluid boundary layers, see, for example, [29, page 129]:

$$
\begin{aligned}
& \dot{x}_{1}=x_{2}, \\
& \dot{x}_{2}=x_{3}, \\
& \dot{x}_{3}=-x_{1} x_{3} .
\end{aligned}
$$

We use the function $h(x)=x_{1}$. Since the plane $\mathscr{P}_{3}$ is invariant, we obtain that any periodic orbit $\Gamma$ is located in the semispace $x_{3}>0$ or in the semispace $x_{3}<0$. So we get that either $\Gamma \cap K_{2}^{+}=\varnothing$ or $\Gamma \cap K_{2}^{-}=\varnothing$. Now we compute that $K_{3}=f^{-1}(0)$. Thus we get that $K_{j}^{+}=\varnothing$ and $K_{j}^{-}=\varnothing$ for $j>3$. Hence the Blasius equations have no periodic orbits.

(2) Even if the matrix $A(x)$ is not given in the triangular form, it is possible in some cases to establish the nonexistence of periodic orbits. We will show how to derive this result in the case of the GMA model for biochemical reactions, see [3, 9].

Firstly, we take the polynomial system (1.1) of the form

$$
\begin{aligned}
& \dot{x}_{1}=l_{1} x_{1}-a_{1} x_{1}^{b_{3}} x_{2}^{b_{1}}, \\
& \dot{x}_{2}=-l_{2} x_{2}+a_{2} x_{1}^{b_{1}} x_{3}^{b_{2}}, \\
& \dot{x}_{3}=-l_{3} x_{3}+a_{3} x_{2}^{b_{1}} .
\end{aligned}
$$

Here, $x_{s}, s=1,2,3$, are concentrations (e.g., of a hormone or a protein), $a_{s}, b_{s}, l_{s}, s=$ $1,2,3$, are some parameters. Let $l_{1} l_{2} l_{3} \neq 0, a_{1} a_{2} a_{3} \neq 0$, and $b_{3} \geq 1$. We notice that $\mathscr{P}_{1}$ is the invariant set. We use the function $h(x)=x_{1}$ and apply Proposition 2.6. It is easy to see that the restriction of system (6.2) on $\mathscr{P}_{1}$ has no periodic orbits. We introduce the set $S_{1}$ defined by the equation $l_{1}-a_{1} x_{1}^{b_{3}-1} x_{2}^{b_{1}}=0$. Then $K_{1}=\mathscr{P}_{1} \cup S_{1}$. Our goal is to compute $K_{3} \cap C\left(\mathscr{P}_{1}\right)$. Applying the Lie derivative to $l_{1}-a_{1} x_{1}^{b_{3}-1} x_{2}^{b_{1}}$, we obtain some polynomial $\eta$, and then we compute the restriction $\left.\eta\right|_{\left\{S_{1}\right\}}$. As a result, we get that $\left.\eta\right|_{\left\{S_{1} \cap C\left\{\mathscr{P}_{1}\right\}\right\}}(x)=0$ if and only if $x_{2}^{b_{1}}\left(l_{2} x_{2}-a_{2} x_{1}^{b_{1}} x_{3}^{b_{2}}\right)=0$. Again, by similar routine computations carried out for the polynomial $x_{2}^{b_{1}}\left(l_{2} x_{2}-a_{2} x_{1}^{b_{1}} x_{3}^{b_{2}}\right)$, we obtain that the set $K_{3} \cap C\left\{\mathscr{P}_{1}\right\}$ is described by the system of equations

$$
\begin{gathered}
l_{1}-a_{1} x_{1}^{b_{3}-1} x_{2}^{b_{1}}=0, \\
l_{2} x_{2}-a_{2} x_{1}^{b_{1}} x_{3}^{b_{2}}=0 \\
l_{3} x_{3}-a_{3} x_{2}^{b_{1}}=0 .
\end{gathered}
$$


So, $K_{3}=f^{-1}(0)$. Now we suppose that the sets $K_{2}^{+}$and $K_{2}^{-}$are crossed by some periodic orbit $\Gamma$. In this case, there are two points $p$ and $q \in \Gamma$ such that

$$
\begin{gathered}
p_{2}^{b_{1}}=l_{1} a_{1}^{-1} p_{1}^{1-b_{3}}, \\
p_{3}^{b_{2}}>l_{1}^{1 / b_{1}} a_{1}^{-1 / b_{1}} l_{2} a_{2}^{-1} p_{1}^{b_{*}}, \\
q_{2}^{b_{1}}=l_{1} a_{1}^{-1} q_{1}^{1-b_{3}}, \\
q_{3}^{b_{2}}<l_{1}^{1 / b_{1}} a_{1}^{-1 / b_{1}} l_{2} a_{2}^{-1} q_{1}^{b_{*}},
\end{gathered}
$$

with $b_{*}=-b_{1}+\left(1-b_{3}\right) / b_{1}$. We notice that (1) if $b_{1}$ is even, $b_{3}$ is odd and $l_{1} a_{1}<0$, or (2) if $b_{2}$ is even and $l_{1} l_{2} a_{1} a_{2}<0$, then (6.4) is not satisfied for $p$ and $q \in \Gamma$. Indeed, in case of (1), real $p_{2}$ and $q_{2}$ do not exist. In case (2), the signs of $p_{1}$ and $q_{1}$ are the same because $\mathscr{P}_{1}$ is not crossed by $\Gamma$. So, the inequalities in (6.4) are always incompatible with $p$ and $q \in \Gamma$. This means that there are no periodic orbits for system (6.2) provided that condition (1) or (2) is fulfilled. Now we suppose that instead of (2) we have (3): $b_{2}$ is a negative rational number, $b_{2}=b_{21} / b_{22}$, where $b_{21} / b_{22}$ is noncancelable and $b_{21}$ is an even numerator. By the same computations, we find that there are no periodic orbits outside the hyperplane $\mathscr{P}_{3}$ provided that one of conditions (1) and (3) is valid.

(3) Consider the mathematical model of the chemical reaction with autocatalytic step, taken from [12]:

$$
\begin{gathered}
\dot{x}_{1}=\gamma-x_{1}-\lambda x_{1} x_{3}, \\
\dot{x}_{2}=x_{1}-x_{2} x_{3}, \\
\dot{x}_{3}=x_{2} x_{3}-\lambda x_{1} x_{3},
\end{gathered}
$$

where $x_{s}, s=1,2,3$, are concentrations of chemicals and $\gamma, \lambda>0$ are parameters. We use the function $h(x)=x_{3}$. Then we introduce the plane $S_{2}$ which is defined by the equation $h_{1}(x):=x_{2}-\lambda x_{1}=0$. We observe that $\mathscr{P}_{3}$ is the invariant plane and we get that each periodic orbit has no common points with $\mathscr{P}_{3}$. Also, $K_{1}=\mathscr{P}_{3} \cup S_{2}$. We obtain that $L_{f} h_{1}=$ $-\gamma \lambda-x_{1}-x_{2} x_{3}+\lambda x_{1}+\lambda^{2} x_{1} x_{3}$. Let the set $S_{3}$ be defined by $h_{1}(x)=L_{f} h_{1}(x)=0$. Then we compute that $S_{3}$ is given by the equations $x_{2}=\lambda x_{1}$ and $\gamma \lambda=(\lambda-1)\left(1+\lambda x_{3}\right) x_{1}$. Also, $\left.L_{f}^{2} h_{1}\right|_{\left\{S_{3} \cap C\left(\mathscr{P}_{1}\right)\right\}}=0$ is given by $\left(\lambda^{2}-\lambda^{4}\right) x_{3}^{2}+x_{3}\left(-2 \gamma \lambda^{3}-2 \gamma \lambda^{2}\right)+\gamma-2 \gamma \lambda^{2}-\gamma \lambda=0$. So, if $\lambda \neq 0, \pm 1$, then the maximal invariant subset in $S_{2}$ can contain not more than two points. One of them is the equilibrium point $\left(\gamma / 2, \lambda \gamma / 2, \lambda^{-1}\right)^{T}$. So, all periodic orbits must cross both of the sets $K_{2}^{+} \cap C\left(\mathscr{P}_{3}\right)$ and $K_{2}^{-} \cap C\left(\mathscr{P}_{3}\right)$. Since $f(x) \neq 0$ for any $x \in \mathscr{P}_{1}$, we deduce that each periodic orbit has no common points with $\mathscr{P}_{1}$. Now let $0<\lambda<1$. Then there are no periodic orbits in $(0, \infty) \times \mathbb{R}^{1} \times(0, \infty) \cup \mathscr{P}_{1} \cup \mathscr{P}_{3}$. In addition, if $\Gamma \subset(-\infty, 0) \times \mathbb{R}^{1} \times$ $(0, \infty)$, or $\Gamma \subset(-\infty, 0) \times \mathbb{R}^{1} \times(-\infty, 0)$, or $\Gamma \subset(0, \infty) \times \mathbb{R}^{1} \times(-\infty, 0)$, then $S_{2}$ is crossed by $\Gamma$. Now let $\lambda>1$. Similarly, there are no periodic orbits in $(-\infty, 0) \times \mathbb{R}^{1} \times(0, \infty) \cup$ $\mathscr{P}_{1} \cup \mathscr{P}_{3}$. In addition, if $\Gamma \subset(0, \infty) \times \mathbb{R}^{1} \times(0, \infty)$, or $\Gamma \subset(-\infty, 0) \times \mathbb{R}^{1} \times(-\infty, 0)$, or $\Gamma \subset$ $(0, \infty) \times \mathbb{R}^{1} \times(-\infty, 0)$, then $S_{2}$ is crossed by $\Gamma$. At last, let $\lambda=1$. Then $\left.L_{f} h_{1}\right|_{\left\{S_{2}\right\}}=-\gamma x_{3}$. Since any periodic orbit $\Gamma$ has no common points with $\mathscr{P}_{3}$, we get that $\left.L_{f} h_{1}\right|_{\left\{S_{2} \cap \Gamma\right\}}$ is a function with only positive or negative values. So, in this case, our system has no periodic orbits in $\mathbb{R}^{3}$. 


\section{A few comments on the localization of periodic orbits}

Information on a location of periodic orbits obtained by our approach crucially depends on a choice of functions $h$ used for the localization. The first important problem consists in the choice of some function $h$ for which one can get the "best possible" localization set of periodic orbits. Here, intuitively, the "best possible" means the smallest set with respect to inclusions. We believe that the clever choice of functions $h$ includes classes of Lyapunov functions, Darboux polynomials, and functions for which the corresponding set $K_{s}=f^{-1}(0)$ for some $s, 1 \leq s \leq n$. However, it may have significance for attempts to localize periodic orbits by using functions $h$ of more general types than mentioned here.

By taking a proper Lyapunov function, it is possible in some cases to localize all periodic orbits in a compact domain, see the example of the Lorenz system in $[1,11]$. Darboux polynomials can provide the localization of periodic orbits in invariant surfaces. However, the latter type of localization is valid only for specific values of parameters of the system. Further, we notice that analysis of sets $K_{j}, j>n$, is not required in our examples, which leads to simple computations. Therefore, it is worthwhile to describe a class of systems for which we can avoid computations of sets $K_{j}, j>n$, by a proper choice of the function $h$.

The localization of periodic orbits by means of their intersections with some surface is easy to obtain, see the computations in our examples. Usually, it has a reasonable value when it is reformulated as a nonexistence condition in the complementary set to this surface.

One more interesting question arising in the process of the localization concerns the idea of "minimizing" in some sense a localization set containing all periodic orbits by a proper choice of a few different functions $h$. For example, if we know that all periodic orbits are located in different sets $U_{1}$ and $U_{2}$ in the state space, then they are contained in $U_{1} \cap U_{2}$ as well. We expect that in this case there is a possibility to play with different Lyapunov functions.

\section{Conclusions}

In this paper, we described sets in the state space of a nonlinear system, which do not contain periodic orbits, contain all periodic orbits, or have common points with any periodic orbit. These sets are given in terms of $f$ and a differentiable, usually polynomial function $h$ used in the process of the localization. Mainly, the case of polynomial systems was examined. It has been established that for a polynomial system and a polynomial function $h$, these sets can be described by means of a finite number $k_{1}\left(k_{2}\right)$ of polynomial equations (polynomial inequalities) appropriately. Upper bounds for $k_{j}, j=1,2$, were provided in different cases. The role of invariant sets in the localization problem of periodic orbits was discussed. Our approach is based on using high-order extremum conditions, high-order tangency conditions of a nonsingular solution with an algebraic set in the state space, and some ideas related to algebraically-dependent polynomials. A few examples were considered including the Blasius equations, the GMA system, and the mathematical model of the chemical reaction with autocatalytic step. Finally, we remark that the results of this paper can be applied to a solution of the localization problem of homoclinic and heteroclinic phase curves. 


\section{References}

[1] A. Babloyantz, A. P. Krishchenko, and A. Nosov, Analysis and stabilization of nonlinear chaotic systems, Comput. Math. Appl. 34 (1997), no. 2-4, 355-368.

[2] N. A. Bobylev, A. V. Bulatov, S. K. Korovin, and A. A. Kutuzov, Limit cycles of autonomous systems, Dokl. Math. 53 (1996), no. 3, 390-393.

[3] R. Díaz-Sierra, B. Hernández-Bermejo, and V. Fairén, Graph-theoretic description of the interplay between non-linearity and connectivity in biological systems, Math. Biosci. 156 (1999), no. 1-2, 229-253.

[4] L. Drossos, O. Ragos, M. N. Vrahatis, and T. Bountis, Method for computing long periodic orbits of dynamic systems, Phys. Rev. E 53 (1996), no. 1, 1206-1211.

[5] V. Franceschini, C. Giberti, and Z. M. Zheng, Characterization of the Lorenz attractor by unstable periodic orbits, Nonlinearity 6 (1993), no. 2, 251-258.

[6] A. Gabrielov and A. Khovanskii, Multiplicity of a Noetherian intersection, Geometry of Differential Equations, Amer. Math. Soc. Transl. Ser. 2, vol. 186, American Mathematical Society, Rhode Island, 1998, pp. 119-130.

[7] W. Grasman, Periodic solutions of autonomous differential equations in higher dimensional spaces, Rocky Mountain J. Math. 7 (1977), no. 3, 457-466.

[8] J. Hofbauer, J. Mallet-Paret, and H. L. Smith, Stable periodic solutions for the hypercycle systems, J. Dynam. Differential Equations 3 (1991), no. 3, 423-436.

[9] D. H. Irving, E. O. Voit, and M. A. Savageau, Analysis of complex dynamic networks with ESSYNS, Canonical Non-Linear Modelling S-Systems Approach to Understanding Complexity (E. O. Voit, ed.), Van Nostrand Reinhold, New York, 1991.

[10] L. Kocarev, U. Parlitz, and B. Hu, Lie deriatives and dynamical systems, Chaos Solitons Fractals 9 (1998), no. 8, 1359-1366.

[11] A. P. Krishchenko, Estimations of domains with cycles, Comput. Math. Appl. 34 (1997), no. 2-4, 325-332.

[12] H. J. Krug and L. Kunhert, Ein oszillierendes Modellsystem mit autokatalytischem Teilschritt, Z. Phys. Chemie 266 (1985), 65-73 (German).

[13] J. P. LaSalle, An invariance principle in the theory of stability, Differential Equations and Dynamical Systems (Proc. Internat. Sympos., Mayaguez, Puerto Rico, 1965), Academic Press, New York, 1967, pp. 277-286.

[14] A. C. Lazer, Topological degree and symmetric families of periodic solutions of nondissipative second-order systems, J. Differential Equations 19 (1975), no. 1, 62-69.

[15] J. Llibre and X. Zhang, Invariant algebraic surfaces of the Rikitake system, J. Phys. A 33 (2000), no. $42,7613-7635$.

[16] Darboux integrability for the Rössler system, Internat. J. Bifur. Chaos Appl. Sci. Engrg. 12 (2002), no. 2, 421-428.

[17] Invariant algebraic surfaces of the Lorenz system, J. Math. Phys. 43 (2002), no. 3, 16221645.

[18] L. Perko, Differential Equations and Dynamical Systems, Texts in Applied Mathematics, vol. 7, Springer-Verlag, New York, 1996.

[19] A. Płoski, On the growth of proper polynomial mappings, Ann. Polon. Math. 45 (1985), no. 3, 297-309.

[20] G. Pólya and G. Szegö, Problems and Theorems in Analysis. Vol. II, Springer-Verlag, New York, 1976.

[21] H. Radjavi and P. Rosenthal, Simultaneous Triangularization, Universitext, Springer-Verlag, New York, 2000.

[22] P. Schmelcher and F. K. Diakonos, General approach to the localization of unstable periodic orbits in chaotic dynamic systems, Phys. Rev. E 57 (1998), no. 3, 2739-2746. 
[23] R. A. Smith, Existence of periodic orbits of autonomous ordinary differential equations, Proc. Roy. Soc. Edinburgh Sect. A 85 (1980), no. 1-2, 153-172.

[24] Certain differential equations have only isolated periodic orbits, Ann. Mat. Pura Appl. (4) 137 (1984), 217-244.

[25] J. C. Sprott, Some simple chaotic flows, Phys. Rev. E (3) 50 (1994), no. 2, part A, R647-R650.

[26] K. E. Starkov, Algebraic aspect of observability property, Automat. Remote Control 55 (1994), no. $12,1754-1761$.

[27] - Some results on periodic orbits of polynomial systems, Stab. Control Theory Appl. 4 (2002), no. 1, 30-41.

[28] Localization/nonexistence conditions of periodic orbits of polynomial systems and their applications, Proc. International Conference on Physics and Control (St. Petersburg, Russia), 2003.

[29] D. J. Tritton, Physical Fluid Dynamics, 2nd ed., Clarendon Press, Oxford University Press, Oxford, 1989.

[30] Y. Q. Ye, S. L. Cai, L. S. Chen, K. C. Huang, D. J. Luo, Z. E. Ma, E. N. Wang, M. S. Wang, and X. A. Yang, Theory of Limit Cycles, Translations of Mathematical Monographs, vol. 66, American Mathematical Society, Rhode Island, 1986.

Konstantin E. Starkov: CITEDI-IPN, avenue del Parque 1310, Mesa de Otay, Tijuana, Baja California 22510, Mexico

Current address: CITEDI-IPN, 2498 Roll Drive 757, San Diego, CA 92154 , USA

E-mail address: konst@citedi.mx 


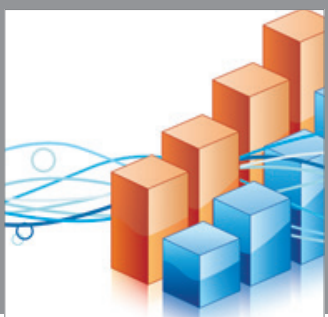

Advances in

Operations Research

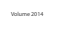

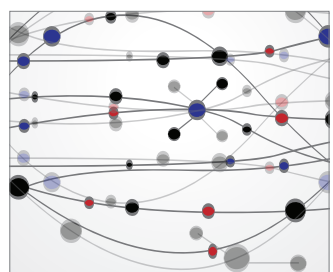

\section{The Scientific} World Journal
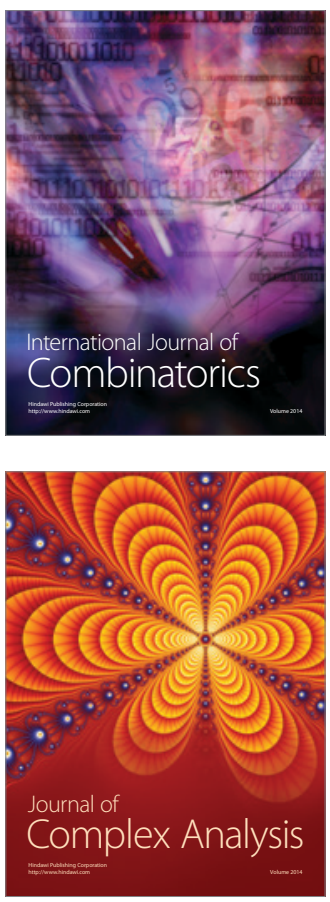

International Journal of

Mathematics and

Mathematical

Sciences
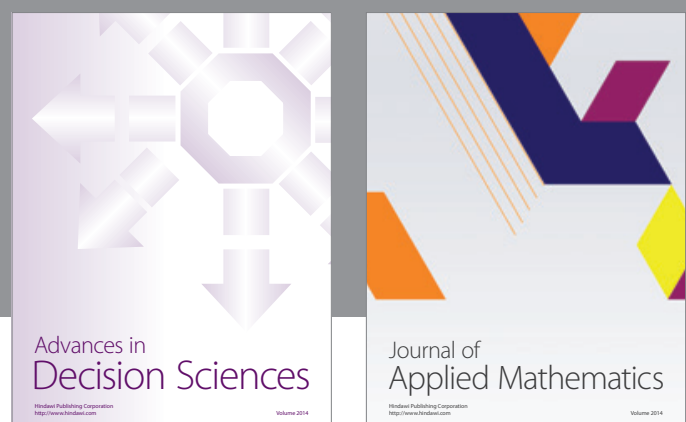

Journal of

Applied Mathematics
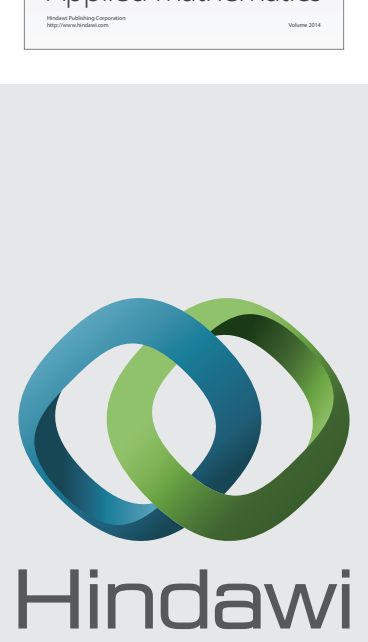

Submit your manuscripts at http://www.hindawi.com
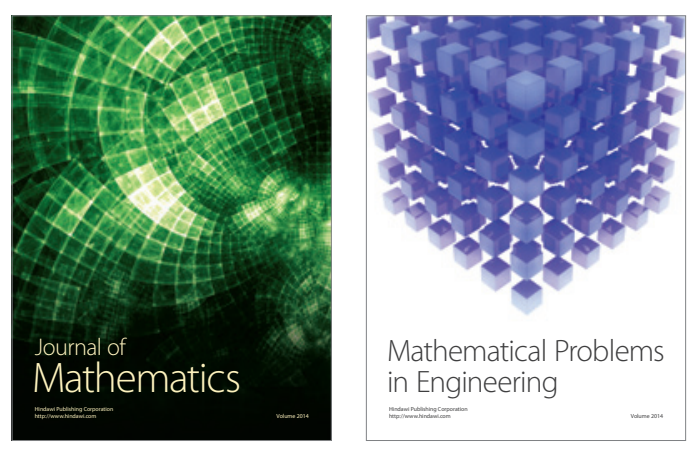

Mathematical Problems in Engineering
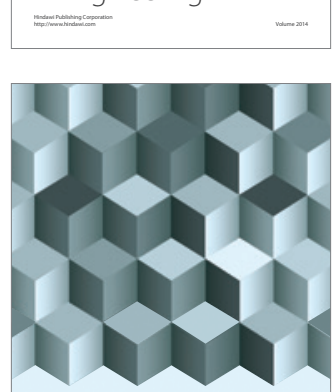

Journal of

Function Spaces
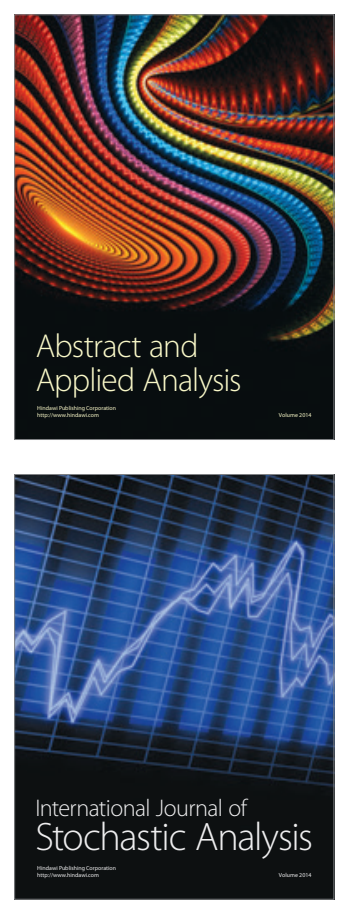

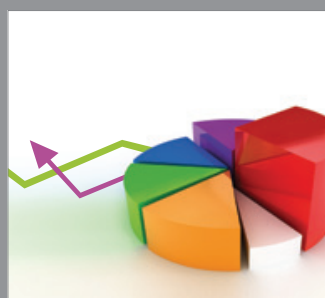

ournal of

Probability and Statistics

Promensencen
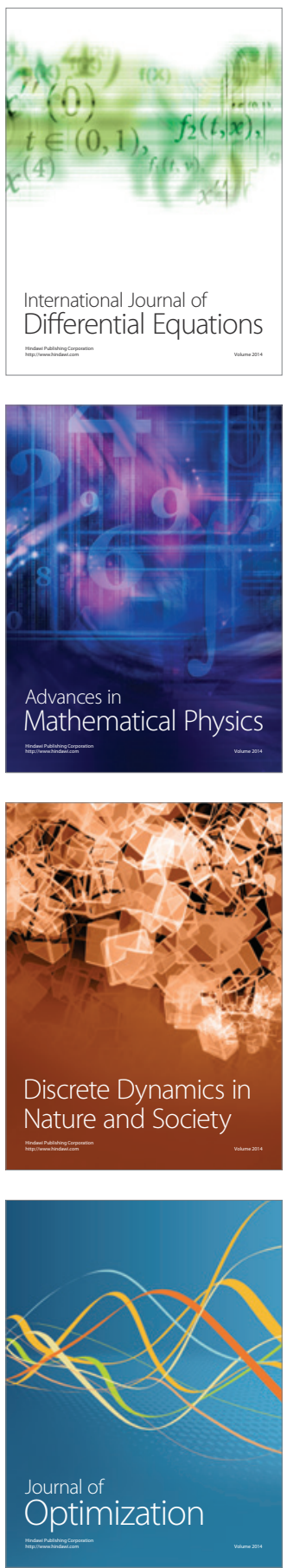